doi: $10.12957 /$ childphilo.2019.42723

\title{
el lugar de la deliberación en la filosofía para niños de lipman
}

\author{
carmina shapiro ${ }^{1}$ \\ universidad nacional de rosario, argentina \\ orcid id: https:/ / orcid.org/0000-0002-3595-976x
}

\section{resumen}

El diálogo ocupa un lugar central en el programa de Filosofía para Niños de Matthew Lipman. Es tanto la actividad central de la Comunidad de Investigación y Cuestionamiento (CI), como el punto de anclaje de la filosofía. Nos interesa aquí revisar en qué sentido la deliberación en la CI es filosófica. En las producciones de Lipman la influencia de Dewey se traduce en una cierta concepción y relación entre democracia, ciudadanía y educación. El presente trabajo, sin embargo, no se centra tanto en la base deweyana de Lipman -cosa que ha sido fructuosamente explorada-, como en la particular articulación que Lipman hace de aquellas nociones para su propuesta de Filosofía para Niños. El modo en que nuestro autor configura la Comunidad de Investigación y Cuestionamiento pone a la Filosofía en un lugar central, pero paradójicamente secundario. Es decir, en la CI la Filosofía se articula con la "democracia como investigación" de tal manera que el rango de posibilidades que le queda a la Filosofía es limitado. Creemos que uno de los factores principales de esta limitación es la concepción deliberacionista del diálogo en la CI. Esto es lo que intentaremos desentrañar aquí, como paso inicial de un trabajo que requerirá sucesivas exploraciones.

palabras clave: filosofía para niños; deliberación; liberalismo; razonabilidad.

\section{the role of deliberation in lipman's philosophy for children}

\begin{abstract}
Dialogue has a central role in Matthew Lipman's program of Philosophy for Children. It is as much the central activity of the Community of Inquiry (CI), as it is the anchor of philosophy. Our interest here is to review in what sense the CI's deliberation is philosophical. In Lipman's work, Dewey's influence leads to a certain conception of and relationship between democracy, citizenship and education. This article, however, does not focus so much on Lipman's Deweyan base -which has already been fruitfully explored- as on the particular articulation that Lipman makes of these ideas for his proposal of Philosophy for Children. The way in which our author configures the Community of Inquiry puts Philosophy in a central, but paradoxically secondary, place. That is, in the CI, Philosophy is articulated with the "democracy as inquiry" in such a way that the scope of possibilities left to Philosophy is limited. We believe that one of the main factors in this limitation is the deliberationist conception of dialogue in the CI. This is what we will try to unravel here, as an initial step in a work that will require successive additional explorations.
\end{abstract}

keywords: philosophy for children; deliberation; liberalism; reasonableness.

1 email: shapiro.carmina@outlook.com

childhood E philosophy, rio de janeiro, v. 15, jun. 2019, pp. 01 - 18

issn 1984-5987 
el lugar de la deliberación en la filosofía para niños de lipman

\section{o lugar da deliberação na filosofia para crianças de lipman}

resumo

O diálogo ocupa um lugar central no programa Filosofia para Crianças de Matthew Lipman. É tanto a atividade central da Comunidade de Investigação e Questionamento (CI) quanto o ponto de ancoragem da filosofia. Nosso interesse aqui é rever em que sentido a deliberação no CI é filosófica. Nas produções de Lipman a influência de Dewey se traduz em uma certa concepção e relação entre democracia, cidadania e educação. O presente trabalho, no entanto, não se concentra tanto na base deweyana do Lipman - que já foi explorada de forma proveitosa -, como na articulação particular que Lipman fez dessas noções para sua proposta de Filosofia para Crianças. A maneira pela qual nosso autor configura a Comunidade de Investigação coloca a Filosofia em um lugar central, mas paradoxalmente secundário. Isto é, na CI a Filosofia é articulada com a "democracia como investigação" de tal maneira que a gama de possibilidades que resta para a Filosofia é limitada. Acreditamos que um dos principais fatores dessa limitação é a concepção deliberacionista do diálogo na CI. É isso que tentaremos desembaraçar aqui, como um passo inicial em um trabalho que exigirá sucessivas explorações.

palavras-chave: filosofia para crianças; deliberação; liberalismo; razoabilidade. 
el lugar de la deliberación en la filosofía para niños de lipman

\section{introducción}

La práctica de la Filosofía para Niños $(\mathrm{FpN})$ ha vivido una expansión intensa, lenta pero constante, en muchos países de Latinoamérica. En ocasiones se la ha asociado a prácticas progresistas o emancipadoras, comparándola por ejemplo, con las prácticas dialógicas de Paulo Freire (Accorinti, 2002; Barrientos Rastrojo, 2013). Los elementos que dan pie a estas relaciones poseen un valor específico dentro del programa, pero si son separados del contexto original de la $\mathrm{FpN}$ y trasladados sin revisión a otros contextos, pueden provocar contradicciones o al menos nudos de difícil solución. Por eso creemos que cobran un nuevo sentido si podemos realmente ver cuál es el fondo epistemológico y político del programa lipmaniano. Cuando escriben sobre estas cuestiones, Lipman y Sharp se posicionan con claridad y cierta coherencia, en tanto los referentes teóricos a los que apelan responden, en rasgos generales, a una misma visión del mundo².

Lipman era un pensador audaz y lo suficientemente meticuloso, y reconocía los problemas de la democracia actual, o al menos algunos de los problemas. Sin embargo, llegado a cierto punto en sus reflexiones, su criticidad alcanza un límite y su visión se percibe muy marcada por su historia como ciudadano estadounidense durante el siglo XX, atravesado por el macartismo, la Segunda Guerra Mundial, la Guerra de Vietnam, la Crisis de los Misiles por nombrar algunos acontecimientos. Digamos que, en Lipman-en-tanto-ciudadano-estadounidense, se perfila un fuerte espíritu liberal y antisoviético, que no siempre resulta patente y no se hace tan evidente en los trabajos más conocidos de la $\mathrm{FpN}$, sino que hay que detenerse y tomarse el tiempo de revisar los fundamentos políticos que no están tan explícitamente trabajados. No son tantos los textos del equipo del IAPC (Institute for

\footnotetext{
${ }^{2}$ Nos referimos a la raíz pragmatista del programa. Si bien hay algunas diferencias entre los autores que refiere Lipman -Dewey y Barber entre otros- y aquellos que refiere Sharp -Rorty y Habermas entre otros-, hay ciertos principios de la práctica que ambos autores sostienen en común. Será tema de un futuro trabajo revisar las diferencias entre ambos.
} 
el lugar de la deliberación en la filosofía para niños de lipman

the Advancement of Philosophy for Children, Nueva Jersey, EUA) que podemos encontrar sobre las cuestiones políticas, y en los escritos no específicos aparecen de una manera un poco desperdigada y difusa. Incluso dentro del marco del mismo programa podemos encontrar unas incongruencias o contradicciones que no son menores, entre lo que se espera que la Filosofía y la comunidad de investigación logren y los fundamentos a partir de los cuales se enuncia.

En este trabajo vamos a centrarnos en reconstruir su idea de democracia y los fundamentos políticos que sostienen a la comunidad de investigación como un valor educativo. La pregunta que vamos a tratar de responder es ipor qué Lipman sostiene que FpN puede ser, no sólo una reforma educativa, sino también una reforma política?, para examinar luego ¿qué lugar configura este marco político-educativo para la filosofía? Lo primero que vamos a hacer entonces es tratar de reconocer los supuestos políticos más fuertes que están operando en el programa de $\mathrm{FpN}$, al tiempo que los relacionamos con la comunidad de investigación.

\section{la reforma educativa como reforma política. democracia como investigación}

Cuando Matthew Lipman elabora su programa de FpN dos de los conceptos más importantes sobre los que se basa son el de comunidad de investigación y cuestionamiento y el de democracia como investigación ${ }^{3}$. Su formación académica en pensamiento crítico y en pragmatismo y, como decíamos más arriba, su perfil de ciudadano estadounidense fueron condicionantes de su formación filosófica, ética y política. Esto dio lugar a cierta concepción de democracia que brinda el marco general del programa, a la vez que resulta condición necesaria e ideal regulativo de la comunidad de investigación. Será condición necesaria porque el maestro debe poner todos sus esfuerzos en generar las condiciones que permitan un diálogo filosófico en esos términos. $\mathrm{Y}$ al mismo tiempo, es ideal regulativo porque funciona como criterio a

\footnotetext{
3 Para la centralidad de la comunidad de investigación y cuestionamiento véase: Lipman, 1997b: 6-21; 1998: 305-321; 2003: 83-104. Para democracia como investigación véase: Lipman, 1992: 305-338; 1998: 322-340; Sharp, 1997: 175-186.
} 
partir del cual se evalúa el crecimiento y desarrollo de estudiantes, comunidad y clases.

Comencemos examinando la cuestión de qué significa para Lipman 'democracia como investigación'. Antes que nada, hay que decir que no es un concepto que encontremos en los escritos de Ann Sharp, y que Lipman no aporta una definición puntual, es decir, no encontramos una sección particular en la que Lipman se dedique a explicar lo que entiende por democracia como investigación. Lo que sí podemos encontrar son los referentes teóricos a los que apela y una particular analogía que emplea para referirse a esto.

La obra de Lipman Thinking in Education, traducida como Pensamiento Complejo y Educación, cuenta con dos ediciones marcadamente diferentes. La primera data de 1991 y la segunda, de 2003, cuando Lipman revisó sus elaboraciones iniciales. Esto le condujo a que, a pesar de conservar algunos capítulos en común, la edición de 2003 sea prácticamente un libro diferente. Nuestro desarrollo se basará en el capítulo 15 de la primera edición de Pensamiento complejo y educación, intitulado "El significado político de la Comunidad de Indagación", puesto que, a pesar de que el mismo desaparece en la edición de 2003, consideramos valioso tomar en cuenta las afirmaciones que Lipman realiza allí, pues es uno de los pocos textos en los que Lipman se explaya con especial atención acerca de los fundamentos políticos del programa.

La primera referencia que aquí aparece es a John Dewey, en su espíritu de lograr una "democracia participativa orientada por la inteligencia" (Lipman, 1998: 332). La inteligencia para Lipman estará profundamente marcada por la razonabilidad, condición principal para realizar buenos juicios, bien fundamentados y sensibles al contexto. Acto seguido, Lipman afirma que este debate nos lleva a Locke más que a la noción de democracia liberal de Rousseau. Semejante afirmación requeriría que Lipman nos explicase qué es lo que entiende por "democracia liberal de Rousseau", puesto que la frase expresa un posicionamiento que no acaba de hacer explícito. Pero sí nos pone una nota al pie. En ella, y fuera del cuerpo del texto, como si se tratara de 
el lugar de la deliberación en la filosofía para niños de lipman

una información lateral, necesaria pero de menor importancia, Lipman hace referencia al historiador Edward Carr quien postula tres enunciados básicos de la democracia moderna, todos ellos presentes en Locke pero presuntamente no en Rousseau:

1) la fuente última de decisión sobre lo que es correcto o incorrecto se halla en la conciencia individual, 2) en las sociedad existe una armonía fundamental de intereses entre los individuos, 3) la discusión racional es el mejor método para poder tomar decisiones en la sociedad. (Lipman, 1998: 332)

Podemos entonces aventurarnos a decir que estos tres elementos van a estar de algún modo presentes en el programa de FpN, en tanto Lipman comparte con Carr la idea de que éstos son tres componentes esenciales de la democracia moderna. Es decir, de alguna manera habríamos de poder ubicar en el programa un cierto trabajo sobre las conciencias individuales, una cierta definición y valoración del tipo de comunicación que constituye un diálogo racional, y un supuesto de muy pocos conflictos de intereses en la sociedad. Al mismo tiempo, estos tres 'enunciados básicos' son perfectamente compatibles con el ideal deweyano de una democracia participativa orientada por la inteligencia.

Unas páginas antes del fragmento aludido, Lipman hace referencia a un libro de 1984 de Benjamin Barber, filósofo político que insiste en que debemos poner nuestros esfuerzos en transformar la actual "democracia liberal" en una "democracia fuerte" (Lipman, 1998: 326-326). Según esto, en las democracias liberales la participación ciudadana se ve restringida al aislamiento del voto, mientras que en una democracia fuerte los ciudadanos toman un papel más activo al participar en las numerosas comunidades públicas que tienden a constituir una comunidad democrática global. La comunidad de investigación y cuestionamiento lipmaniana (CI) se establecería, entonces, como una de esas instancias intermedias de participación. Una vez más, encontramos que hay coherencia entre este planteo y uno de los supuestos deweyanos que Lipman retoma, que concibe al aula como un microcosmos que refleja a la Gran Comunidad (Lipman, 1998: 339). En concordancia con esta creencia deweyana, va a sostener Lipman que: 
la comunidad de investigación representa la dimensión social de la práctica democrática y la garantía de su implantación, así como del desarrollo pleno de sus potencialidades. Por otro lado, la dimensión cognitiva de la práctica democrática viene representada por el proceso de investigación que ha sido identificado como la transición de la deliberación al juicio. (Lipman, 1998: 329).

Es decir, Lipman afirma convencido que hay una dimensión deliberativa, una dimensión social y una dimensión cognitiva de la práctica democrática que se concretan en la comunidad de investigación y cuestionamiento. La CI lleva adelante un proceso de investigación que consiste en la transición de la deliberación al juicio (dimensiones deliberativa y cognitiva), proceso que siempre es realizado con pares (dimensión social). De este modo es que Lipman sostiene que la comunidad de investigación y cuestionamiento puede ser considerada como “intersección embrionaria entre democracia y educación" (Lipman, 1998: 329).

¿Cómo habrían de ser estos ciudadanos ideales, democráticos y orientados por la inteligencia? Indirectamente, encontramos una caracterización general en la descripción que hacen Lipman y Sharp de la comunidad de investigación y cuestionamiento "madura". Como delineación incipiente valga mencionar la expresión de Ann Sharp en su artículo "La comunidad de cuestionamiento e investigación filosóficos: educación para la democracia", que sostiene que:

Los alumnos parecen rechazar el rol de prima donna y se muestran capaces de colaborar y cooperar en la investigación. Pueden escuchar y recibir lo que otros tienen para decir, de tal modo que el sentido y la vitalidad son compartidos. Se liberan de la necesidad de tener siempre la razón. Tienen el coraje y la capacidad de cambiar de opinión y de sostener sus opiniones de manera tentativa. (Sharp, 1997: 178).

En esta descripción podemos ver que la filosofía está valorizada solamente en tanto posibilita el camino hacia ese ideal ciudadano.

\section{el modelo jurídico como modelo de búsqueda de la verdad y de investigación correcto}

Revisemos ahora su recurso a la analogía jurídica para intentar discernir un

poco más qué es lo que quiere implicar Lipman cuando propone buscar una democracia como investigación. Tres fragmentos resultarán especialmente 
significativos en este intento de sopesar las consecuencias filosóficas que esta analogía supone sobre la comunidad de investigación y cuestionamiento. En los tres casos la referencia viene a colación de pensar la investigación, que es jurídica en la analogía, y es filosófica en la CI. ¿Qué es entonces lo que hay en común entre ambas actividades? Como veremos a continuación, de algún modo Lipman está considerando que el tipo de actividad que se desarrolla en una CI es una deliberación. Este sería la primera semejanza que podemos establecer entre ambos elementos de la analogía.

En Pensamiento Complejo y Educación de 1991, hallamos una mención a esta analogía en el capítulo titulado "El significado político de la comunidad de investigación", que la relaciona explícitamente con la idea de democracia deliberativa.

Pero creo que la noción de democracia como investigación conecta a su vez la racionalidad con el consenso y esta conexión es superior a la orientación por dichos criterios aislados. Seguramente el razonamiento judicial no será demasiado aplicable a otras instituciones, pero implica una división del trabajo en la que el juez experto de la ley queda compensado por el jurado con sentido común, y ambos a su vez equilibrados por los juristas preparados para sus discursos retóricos sobre la acusación y la defensa. Todos se hallan implicados en un proceso de investigación y su meta es el logro de resultados razonables. (Lipman, 1998: 324)

Si bien encontramos una incipiente caracterización de los roles de cada una de las figuras jurídicas, nos centraremos de momento en otra cuestión. De este fragmento nos interesa destacar la valoración que Lipman hace de la división de tareas, según la cual los roles no se solapan porque cada uno hace su aporte específico, mientras colaboran investigando conjuntamente para llegar a un fin común que es el logro de resultados razonables.

Un capítulo antes, sin embargo, encontramos un fragmento aún más relevante, en el capítulo 14 "Pensamiento y comunidad" de la edición de 1991 de Pensamiento Complejo y Educación, subtitulado "El jurado como ejemplo de diálogo deliberativo" (pp. 316-319). Este breve texto, aunque desaparece en la revisión de 2003, se vuelve particularmente pertinente a los fines del presente análisis, ya que es uno de los fragmentos más específicos que podemos encontrar en Lipman sobre el tema. En una 
entrevista de 1996 que Walter Kohan le realiza a Lipman, éste todavía menciona la comparación con los procedimientos jurídicos, por lo que examinar cuáles son las razones que llevan a Lipman a abandonar esta sección en 2003, revisando a la vez si abandonar la sección y la analogía implicó abandonar los supuestos que las sostienen, es un punto de fuerte interés pero será motivo de un futuro trabajo.

En este fragmento es donde Lipman pone más detalle a la función que cumple cada figura dentro del marco de la investigación jurídica. Del jurado va a decir que expresa el razonamiento común, que debe ser consciente de pruebas y evidencias, que es autocorrectivo, que se ocupa de examinar las versiones y llegar a una "comprensión única de las evidencias y de los testimonios" (Lipman, 1998: 316) expresada en el veredicto, y que su objetivo no es persuadir sino ser sensible al contexto. El jurado tomará la información legal específica que brinde el juez para realizar las inferencias que el caso amerite según se ajuste con mayor o menor precisión a la ley (Lipman, 1998: 317).

La participación que sí resulta de talante persuasivo es la de los abogados o juristas, que presentarán argumentos agonistas, contrarios y contenciosos, originales e ingeniosos, pero siempre unilaterales (Lipman, 1998: 316).

Del juez dice Lipman que 'hila fino en la ley' en tanto es un experto en ella, ocupándose de brindar el marco legal pertinente para que el jurado pueda elaborar un buen veredicto. Luego, aplica principios legales y principios lógicos como criterios para elaborar sus juicios, utilizando procedimientos deductivos (Lipman, 1998: 317). Esta caracterización da a entender que el juez no será quien se ocupe de hacer estimaciones particulares sobre el caso, sino que su rol se desempeña dentro del marco de las relaciones lógicas que implica la ley. Al ponerlo en estos términos, Lipman está también insinuando que la tarea de un juez es principalmente lógica y que sus decisiones se derivan de los análisis de carácter lógico-jurídicos que realice, análisis en los que no serían relevantes ni influyentes sus creencias personales, ni sus convicciones acerca del mundo. $\mathrm{O}$ en otras palabras, siguiendo a Kohan y Waksman 
(2000: 60), el aspecto ético de la labor del juez queda supeditado al aspecto lógico, que viene primero y resulta principal.

En este punto de la analogía, parece que podemos tomar dos caminos para su interpretación. Uno de los caminos posibles es relacionar los tres personajes del campo jurídico -jurado, abogados y juez- con los roles de las personas en una comunidad de investigación y cuestionamiento. De acuerdo con la descripción revisada, entonces, la figura del juez podría resultar equivalente con la figura del docente filósofo en la CI, en tanto ambos son expertos en cada campo y saben utilizar minuciosamente los procedimientos lógicos. En principio, esta equivalencia es la más sencilla de establecer. Sin embargo, se vuelve un poco más complicado continuar: ¿con qué homologamos a los estudiantes y a la comunidad? Podemos aventurarnos a afirmar que los estudiantes cumplen una función semejante a la del jurado, representando el sentido común y la sensibilidad al contexto. También son los estudiantes en el aula los "encargados" de llevar adelante el diálogo en la CI, lo que podría corresponder con la labor del jurado en un juicio en tanto dialogan para llegar a un veredicto. $\mathrm{Y}$ aquí es donde nos topamos con uno de los límites de establecer la analogía de esta manera. ¿Podemos afirmar que la comunidad de investigación y cuestionamiento sería equivalente al hacer de los abogados, cuyos argumentos "fueron construidos cuidadosamente y son a menudo originales e ingeniosos, pero son siempre unilaterales" (Lipman, 1998: 316)? No parece de ningún modo adecuado. Se espera que la CI sea capaz de detectar los errores argumentales y en consecuencia autocorregirse, pero no podemos aceptar que el diálogo filosófico esté regido por la confrontación y la intención de convencer a los otros. Si en cambio dijéramos que el jurado y su diálogo se corresponden con la CI, ¿qué rol cabría a los abogados? Tal vez podríamos decir que cada participante de la CI sería como un abogado defendiendo su punto de vista y su argumento, y contrariando a las otras perspectivas. Pero esto iría en contra del ideal de apertura mental (open mindness) que caracteriza a los participantes de una CI madura. 
Otro camino de interpretación posible es establecer la relación de analogía entre los personajes de la investigación jurídica y las facultades intervinientes en una investigación filosófica ideal en términos lipmanianos. En estos términos, podríamos relacionar a cada uno de los personajes con las actividades dialógicas y de razonamiento que realiza cada participante de la CI. Es decir, la analogía no describiría tanto la organización de una comunidad, sino más bien lo que ocurre en la mente de cada participante, como si fueran facultades que se ponen en juego al interactuar en la CI. Así planteado, podríamos estimar que el jurado representa las opiniones preconcebidas, el conjunto de las experiencias vividas, y los razonamientos prácticos cotidianos que no buscan más que atender a las necesidades de la vida. El juez representaría el buen proceder lógico que conoce los alcances y relaciones de cada concepto (recordemos que Lipman describe al juez como un experto en su campo). Podría ser interesante concebirlo como facultad de metacognición, aunque tal vez sea decir demasiado. Por último, los abogados representarían la capacidad de construir argumentaciones que apoyen adecuadamente cada punto de vista.

Ya que Lipman afirma que las tres figuras jurídicas trabajan con un interés común hacia un mismo fin, podríamos pensar que estas facultades se combinan para llegar a un juicio adecuado, sea realizado individual o intersubjetivamente. Pero es sólo de la combinación de la actividad de las tres figuras que es posible que surja el pensar de orden superior. El juez sin los otros dos no sería más que una suerte de técnico de la ley, o un diccionario de conceptos y relaciones sin ningún vínculo con la experiencia porque su esquematicidad se vería constantemente sacudida y superada por la complejidad de las vivencias. El jurado sin los otros dos no sería más que el vivir sensible a las circunstancias pero un poco contradictorio y poco preciso en sus afirmaciones. Los abogados sin los otros dos no serían más que una suerte de monólogos batallantes, sólidos y convincentes pero cerrados e inflexibles. Del trabajo cooperativo entre estas tres figuras es que surge el buen pensar, lógico, sensible al contexto y prolijo en sus definiciones. 
Nos resulta difícil tomar partido por una de estas dos posibilidades; Lipman mismo no desarrolla mucho más este asunto. En consecuencia, creemos que bien vale sostener ambas lecturas de la analogía, reconociendo los aportes y dificultades de cada una, porque ese ejercicio de discernimiento finalmente nos lleva a pensar de nuevo la comunidad de investigación y cuestionamiento, y en el proceso de llegar a las conclusiones es que encontramos su valor. Por eso, preferimos no clausurar las dificultades y alternativas y dejarlas abiertamente planteadas.

\section{deliberar no es filosofar}

Abriendo las miras a un análisis un poco más general, queremos destacar dos cuestiones que Lipman valora del sistema de jurado. Una de estas cuestiones es que el mismo no requiere ninguna prueba de razonamiento ni evaluación de aptitud para que cualquier ciudadano pueda formar parte, sólo se requiere que no haya un interés personal que parcialice su perspectiva sobre el caso (Lipman, 1998: 318). Esta observación resulta interesante en la analogía porque nos habla de la capacidad que todos tendríamos de mejorar nuestra manera de pensar, con el solo hecho de tener tiempo para la deliberación y un grupo de personas "imparciales" que pueda investigar colaborativamente en comunidad. $\mathrm{Si}$ bien sabemos que en nuestras sociedades capitalistas disponer del tiempo a discreción personal es casi un lujo que excede a los sectores sociales más desaventajados, esta observación de Lipman no deja de ser un posicionamiento interesante en el momento en que sostiene que el marco que puede dar ese tiempo para la deliberación a los estudiantes es la comunidad de investigación y cuestionamiento en el aula. Es una afirmación muy significativa el dar pie a esta posibilidad y proponer y defender la CI como un espacio donde se puede practicar el diálogo libremente en la escuela, siguiendo la conversación y la reflexión hacia donde lleve.

La otra cuestión que queremos destacar, y traer con toda su conflictividad, es que el jurado debe "examinar estas diferentes versiones de lo acontecido y llegar, a través de una deliberación, a una comprensión única de las evidencias y de los 
testimonios [...] para hallar un veredicto" (Lipman, 1998: 316). Esta tarea es clara en el marco del sistema de jurado, pero no resulta ni tan clara ni tan evidente en el diálogo en comunidad. ¿Es uno de lo objetivos de FpN llegar a una comprensión única de las cosas? Tanto Lipman como Sharp son pensadores perspicaces y saben que exigir semejante cosa es insostenible en los hechos y hasta podría resultar anti-filosófico, pero sí confían en la construcción de consensos, al menos provisorios. Subyace, de algún modo, a FpN la creencia de que la democracia como consenso deliberativo es un punto límite de toda discusión, punto de acuerdo general que no es puesto en cuestión. Subyace, de algún modo, el supuesto de que toda persona razonable tendrá una preferencia casi natural por la democracia entendida como consenso y deliberación. Su confianza en esto no quita que sea problemático, primero porque aunque Lipman reconoce el poder destructivo del capitalismo, no acaba de problematizarlo al no considerar las relaciones de poder que condicionan a la democracia real (Kohan, 2018), y segundo porque nos obliga a reconsiderar cuál es el lugar de la filosofía en FpN.

En este mismo fragmento de Pensamiento Complejo y Educación de 1991, Lipman cita un párrafo de Michael Walzer que nos ayuda en la tarea de comprender el lugar que Lipman asigna a la analogía jurídica. La cita nos parece relevante para sostener la afirmación de que el gesto de introducir la analogía expresa algo acerca de la P4C que va más allá del hecho de que la analogía sea en efecto introducida:

Apoyamos el sistema del jurado porque creemos que los jurados desinteresados se acercarán más a la verdad. Entonces, ¿por qué no extenderlo a todos los modelos de búsqueda de la verdad y de investigación correctos? Incluso un buen número de filósofos contemporáneos abogarían por la sustitución del debate político por una visión idealizada de las deliberaciones judiciales. [Fin de cita en el original.] ((1989-90) 'A Critique of Philosophical Conversation', Philosophical Forum, 21:1-2, 195n.). (Lipman, 1998: 318)

En este párrafo creo que podemos encontrar el espíritu que lleva a Lipman a trabajar esta analogía, esto es, pensar al sistema del jurado como modelo de búsqueda de la verdad y de investigación correctos. Es llamativo que en todos estos desarrollos no aparece la filosofía mencionada en primer lugar, sino que emerge a colación de la 
el lugar de la deliberación en la filosofía para niños de lipman

pregunta acerca de cómo lograr que los niños sean más razonables y piensen mejor. Llama también la atención que los términos que utilizan tanto Lipman como Splitter y Sharp para hablar de lo que se hace en la CI sean 'deliberar' y 'deliberación'. ¿Qué lugar ocupa y qué sentido tiene la filosofía en el esquema?

El modelo del jurado es un modelo de deliberación. El producto o el resultado de una deliberación es la elaboración de un juicio. La RAE define deliberar de la siguiente manera: "Del lat. deliberāre. 1. intr. Considerar atenta y detenidamente el pro y el contra de los motivos de una decisión, antes de adoptarla, y la razón o sinrazón de los votos antes de emitirlos. 2. tr. Resolver algo con premeditación." (RAE, http://dle.rae.es/srv/fetch?id=C7FSCvp, 27/09/2018). Splitter y Sharp dicen del juicio que "está implicado siempre que elegimos entre cursos de acción en conflicto, o reflexionamos sobre la opinión o el punto de vista de alguien (como cuando juzgamos que lo que dijo es verdadero o falso)" (Splitter y Sharp, 1996: 30). Ambas ideas están relacionadas con el proceso de toma de decisiones o, para decirlo de modo más general, proceso de posicionamiento en torno a una cuestión.

Si el objetivo general de FpN es formar ciudadanos razonables y democráticos, entonces debe de algún modo proveer a los niños de criterios para que las decisiones o posiciones que tomen sean más razonables y democráticas, o debe al menos acompañar a los niños en el proceso de formar esos criterios. Resulta entonces que la elección de criterios por parte de los niños está atravesada por la expectativa de que se transformen en adultos razonables y democráticos. A este respecto, Splitter y Sharp afirman que, en tanto una educación con fines democráticos debe formar para el juicio, la filosofía es la disciplina indicada para desarrollar en los niños la capacidad, la inclinación y la sensibilidad para aprender a partir de lo que experimentamos.

La indagación deliberativa basada en la experiencia y guiada por criterios es el proceso que subyace a la formación del juicio. Como tal, es un proceso que ayuda a cimentar la propuesta a favor de incluir a la filosofía en el corazón del currículum, pues a la par que es verdad que cada disciplina tiene su porción de temas discutibles e intrigantes que requieren del juicio razonado, sólo la disciplina de la filosofía es idónea para escudriñar cuestiones que conciernen a las interconexiones entre la experiencia, los criterios y el juicio. En 
consecuencia, la filosofía constituye un marco disciplinario apropiado para el fortalecimiento del juicio. (Splitter y Sharp, 1996: 33)

Esta afirmación de los autores sugiere sutilmente que la filosofía parece acoplarse a un proyecto de trabajo que preexiste a $\mathrm{FpN}$, y para el cual no queda del todo justificado cuál es el valor de la filosofía en el mismo. Resulta relevante en este punto recordar las críticas que Kohan realizó al modo en que Lipman concibe al pensar filosófico, como habilidad, con jerarquías y moralizado (Kohan, 2004: 119-122). No parece exagerado afirmar que la filosofía queda recortada en FpN, sólo convocada en tanto puede hacer un aporte -central, es cierto, pero sin duda sesgado- al servicio de un proyecto y unos ideales que no son de ningún modo los únicos que la filosofía admite. El recorrido que las novelas realizan por la historia de la filosofía se centra en aquellos planteos filosóficos que contribuyen al ideal preestablecido de formar ciudadanos razonables y democráticos. Y la falta de reconocimiento por parte de Lipman de su propia perspectiva teórica, con sus potencialidades y sus límites, provoca el riesgo de que una concepción particular de la filosofía sea difundida como la única (Kohan y Waksman, 2000: 56-57). En una futura investigación intentaremos estimar qué sucedería con el programa de Lipman si quitáramos a la filosofía del corazón del programa, qué otras relaciones se verían modificadas en consecuencia y qué conceptos serían difíciles de sostener.

De un modo similar al de la cita de Splitter y Sharp, en una conferencia dictada en 1998, Lipman afirma explícitamente que los mecanismos mediante los cuales se puede "sustituir la discusión, entre aquellos que sostienen posturas contrarias, por la comprensión de que la deliberación es investigación" (Lipman, 2016: 83), son los mismos mecanismos que operan cuando la investigación filosófica se lleva a cabo en la escuela primaria. Esto implicaría un elemento de semejanza entre la democracia y la filosofía, según el cual Lipman hace coincidir la investigación en el marco de la democracia con la investigación en el marco de la filosofía, en tanto ambas son actividades de deliberación. Pero en este punto es imprescindible preguntarnos, ¿la filosofía es deliberación? Detendremos aquí este trabajo, pero no sin antes dejar constancia de que el camino a seguir será revisar detenidamente la caracterización 
el lugar de la deliberación en la filosofía para niños de lipman

que Lipman y su equipo hacen de la filosofía, para luego contrastarla con el marco político que él mismo configura para $\mathrm{FpN}$, analizando si coinciden ambas formulaciones y perspectivas.

\section{comentarios finales}

Teniendo en cuenta el espíritu de reforma educativa que Lipman anuncia para su FpN, al encarar la lectura de un capítulo intitulado “El significado político de la Comunidad de Indagación", esperábamos encontrar un análisis de los supuestos filosóficos que lo mueven, con una revisión de las consecuencias políticas de la práctica filosófica y su potencial impacto en las relaciones en el aula o en la educación. En cambio, nos encontramos con un capítulo con un tono mucho más práctico que de examen, que no se diferencia tanto del tono de tantos otros capítulos referidos a otros temas del programa. La ausencia de ciertas definiciones o concepciones claves, como las de 'democracia como investigación', 'institución' o 'política', obtura un poco la posibilidad que tendríamos de discutir esas definiciones, analizar su relación con otros supuestos de $\mathrm{FpN}$ y buscar eventualmente otras que se adecuen mejor a la propuesta. En consecuencia, su visión particular de estos asuntos queda plasmada como una visión que cualquier lector compartiría, casi como si fuera obvio o natural que coincidiéramos en los supuestos que le sostienen, y por eso no fuera necesario explicitarlos ni discutirlos. Parece rondar algo parecido a la expectativa que mencionábamos más arriba de que cualquier persona razonable tendría una preferencia casi natural por la democracia liberal deliberativa.

Sin embargo, creemos que estas mismas omisiones expresan un posicionamiento. Como vimos, la filosofía aparece en el programa condicionada y de alguna manera subordinada a los objetivos socio-políticos del mismo. Es importante aclarar que ubicar a la filosofía en unos ideales políticos no es en sí mismo el problema, sino que el recorte y selección histórico-curricular que FpN hace de la filosofía la dejan perfilada como una mera herramienta, como un corpus que sólo cobra valor en la medida en que puede aportar a esos objetivos socio-políticos un 
orden de disciplinamiento del pensar (Kohan, 2004). “Si la filosofía no llevase a los niños a ser personas democráticas o si ellos pudiesen llegar a ser así formados de otra manera, no habría necesidad de la filosofía en la escuela. La enseñanza de la filosofía se inscribe así en una lógica de educación formadora según una ética y una politica ya determinadas, anteriores, guiadoras, fundantes. La filosofía sirve al sueño democrático de Lipman." (Kohan, 2004: 1256)

Creemos que uno de los motivos que explican la ausencia de estas definiciones y explicitaciones es la filiación de Lipman con la tradición pragmatista liberal y la visión rawlsiana de justicia como equidad, visión que precisamente plantea que tales definiciones no deberían formar parte del juego político porque no admiten ser consideradas ni generales ni públicas. Y Rawls denomina a esas definiciones con el epíteto de "filosóficas" y "metafísicas". Nos llama la atención este punto de semejanza entre Lipman y Rawls, y creemos que continuar el análisis por esta línea puede contribuir aún más a discernir cuál es el real lugar que ocupa la filosofía en el esquema de reforma educativa y política de $\mathrm{FpN}$.

\section{bibliografía}

ACCORINTI, Stella. (2002) “Matthew Lipman y Paulo Freire: Conceptos para la libertad" In: Utopía y Praxis Latinoamericana, vol. 7, núm. 18, septiembre, 2002, pp. 35-56. Universidad del Zulia, Maracaibo, Venezuela.

BARRIENTOS RASTROJO, José (Editor). (2013) Filosofía para niños y capacitación democrática freiriana. Madrid: Liber Factory.

KOHAN, Walter O. y WAKSMAN, Vera. (2000) Filosofía con niños. Aportes para el trabajo en clase. Buenos Aires: Ediciones Novedades Educativas.

KOHAN, Walter O. (2004) Infancia: entre educación y filosofía, Buenos Aires: Laertes.

KOHAN, Walter O. (1997) “Conversaciones con J. Dewey y M. Lipman”. In: KOHAN, Walter O. y WAKSMAN, Vera (Orgs.) ¿Qué es Filosofía para Niños? Ideas y propuestas para pensar la educación, pp. 401-409. Buenos Aires: Oficina de Publicaciones del CBC, EUDEBA.

KOHAN, Walter O. (2018) "Paulo Freire and Philosophy for Children: A Critical Dialogue". Studies in Philosophy and Education, 37(6): 615-629. https://doi.org/10.1007/s11217018-9613-8

LIPMAN, Matthew. (1997a) "Personas No Razonables, Juicios Inapropiados". In: KOHAN, Walter O. Y WAKSMAN, Vera (Orgs.) ¿Qué es Filosofía para Niños? Ideas y propuestas para pensar la educación, pp. 247-258. Buenos Aires, Argentina: Oficina de Publicaciones del CBC.

LIPMAN, Matthew. (1997b) “Thinking in Community”. Inquiry, vol. 16, no. 4, pp. 6 - 21. 
el lugar de la deliberación en la filosofía para niños de lipman

LIPMAN, Matthew. (1991/1998) Pensamiento complejo y educación. Ed. de la Torre, Madrid. LIPMAN, Matthew. (2003) Thinking in education. Cambridge University Press, Cambridge. LIPMAN, Matthew. (2016) El lugar del pensamiento en la educación. Barcelona: Octaedro.

LIPMAN Matthew, SHARP Ann, OSCANYAN Frederick. (1992) La filosofía en el aula. Madrid: De La Torre.

RAE, Disponible en: <http:/ / dle.rae.es/srv/ fetch?id=C7FSCvp> Consulta: 27/09/2018.

SHARP, Ann M. y SPLITTER, Laurance J. (1996) La otra educación. Filosofía para niños y la comunidad de indagación. Buenos Aires: Manantial.

SHARP, Ann Margaret. (1997) “La comunidad de cuestionamiento e investigación filosóficos: educación para la democracia". In: KOHAN, Walter O. Y WAKSMAN, Vera (Orgs.) ¿Qué es Filosofía para Niños? Ideas y propuestas para pensar la educación, pp. 175-186. Buenos Aires, Argentina: Oficina de Publicaciones del CBC.

recibido en: 15.05 .2019

aceptado en: 30.05 .2019 